\title{
Degeneracy and stability in neural circuits of neuromodulators
}

\author{
Chandan K. Behera ${ }^{1}$, Alok Joshi ${ }^{1}$, Da-Hui Wang ${ }^{2,3}$, Trevor Sharp ${ }^{4}$ and KongFatt Wong-Lin ${ }^{1, *}$ \\ ${ }^{1}$ Intelligent Systems Research Centre, School of Computing, Engineering and Intelligent \\ Systems, Ulster University, Derry Londonderry, Northern Ireland, UK \\ ${ }^{2}$ State Key Laboratory of Cognitive Neuroscience and Learning, Beijing Normal University, \\ Beijing, China \\ ${ }^{3}$ School of Systems Science, Beijing Normal University, Beijing, China \\ ${ }^{4}$ Department of Pharmacology, University of Oxford, Oxford, UK
}

\section{${ }^{*}$ Corresponding Author}

KongFatt Wong-Lin

Address: Intelligent Systems Research Centre, Ulster University, Northland Road, Derry Londonderry, BT48 7JL, Northern Ireland, UK

Tel: +44 (0)28 71675320

E-mail: k.wong-lin@ulster.ac.uk

\section{Acknowledgements}

CKB was supported by Ulster University Research Challenge Fund. AJ, TS and KFW-L were supported by BBSRC (BB/P003427/1). KFW-L and D-HW were jointly supported by The Royal Society - NSFC International Exchanges. KFW-L was further supported by COST Action Open Multiscale Systems Medicine (OpenMultiMed) supported by COST (European Cooperation in Science and Technology), and Northern Ireland Functional Brain Mapping Facility (1303/101154803) funded by Invest NI and the University of Ulster. D-HW received further support by NSFC under grants 31671077 and 31511130066. 


\begin{abstract}
Degenerate neural circuits perform the same function despite being structurally different. However, it is unclear whether neural circuits with interacting neuromodulator sources can themselves be degenerate while maintaining the same neuromodulator function. Here, we address this by computationally modelling the neural circuits of neuromodulators serotonin and dopamine, local glutamatergic and GABAergic interneurons, and their possible interactions, under reward/aversion-based conditioning tasks. We show that a single sparsely connected neural circuit model can recapitulate many separate experimental findings, but not all, suggesting multiple parallel circuits. Using simulations and dynamical systems analysis, we demonstrate that several different stable circuit architectures can produce the same observed network activity profile. Further, simulating dopamine (D2) receptor agonists in rewarding task can distinguish among sub-groups of these degenerate networks; a testable model prediction. Overall, this work suggests the plausibility of degeneracy within neuromodulator circuitry and has important implication for the stable and robust maintenance of neuromodulator functions.
\end{abstract}


The nervous system can be modulated by a group endogenous chemical messengers, often called neuromodulators (Kaczmarek and Levitan, 1987). Neurons or synapses, and hence neural circuits, that succumb to neuromodulation often change circuit configuration and function (Marder, 2012; Marder et al., 2014). However, neural circuits can also be degenerate, that is, circuits consisting of different elements and/or structure while performing the same function or yielding the same output (Cropper et al., 2016). This can allow robust maintenance of functions and behaviour in the face of changes in the underlying structure (Edelman and Gally, 2001; Whitacre, 2010). Although it has been shown that neuromodulators can selectively regulate degenerate neural circuits (Marder et al., 2014; Cropper et al., 2016), it is unclear whether neural circuits with neuromodulator-containing neurons can themselves be degenerate, which could in turn provide stable widespread neuromodulator influences on targeted neural circuits (Fig. 1a).

In this theoretical study, we investigate the plausibility of degenerate and stable neuromodulator circuits by focusing on the neural circuits in the midbrain which are the source of ascending pathways of two highly studied monoaminergic neuromodulators, serotonin (5hydroxytrptamine; 5-HT) and dopamine (DA). These neuromodulators have major roles in modulating various cognitions, emotions and behaviours, and are linked to the pathogenesis and pharmacological treatment of many common neuropsychiatric and neurological disorders (Müller and Cunningham, 2020). The majority of 5-HT-producing neurons are found in the dorsal and median raphe nuclei (DRN and MRN), while most DA-producing neurons reside in the ventral tegmental area (VTA) and substantia nigra compacta (SNc) (Müller and Cunningham, 2020). Importantly, there is increasing evidence for direct synaptic interactions between these two neuromodulators, particularly at the level of the VTA (Di Giovanni et al., 2008; Boureau and Dayan, 2011; De Deurwaerdère and Di Giovanni, 2017).

At the functional level, DA and 5-HT are known to play a critical role in reward and punishment (Hu, 2016). For example, there is strong evidence that DA neuronal activity signals reward prediction error (difference between predicted and actual reward outcome) to guide reinforcement learning (Doya, 2002). Specifically, DA neurons are phasically excited upon unexpected reward outcome or reward-predictive cues, and inhibited upon unexpected reward omission or punishment (Schultz et al., 1997; Cohen et al., 2012; Watabe-Uchida et al., 2017), although there is heterogeneity amongst DA neurons in this regard (Cohen et al., 2012; Lammel et al., 2014; Morales and Margolis, 2017; de Jong et al., 2019).

In comparison to DA neurons, DRN 5-HT neurons exhibit greater complexity in function, with recent studies reporting that $5-\mathrm{HT}$ neuronal activity encodes both reward and punishment. For 
instance, Cohen et al. (2015) found that certain 5-HT neurons (labelled "Type I") were phasically activated only by reward predicting cues, but not punishment in a classical conditioning paradigm. On the other hand, another population of 5-HT neurons ("Type II") signalled both expected reward and punishment with sustained elevated activity towards reward outcome (Cohen et al., 2015). The latter study also found that baseline firing of TypeI 5-HT neurons was generally higher in rewarding than punishment trials, and this effect lasted across many trials, suggesting information processing over a long timescale. Moreover, DA neurons did not exhibit this property. Similar 5-HT neuronal responses to reward and punishment were reported in other rodent (Liu et al., 2014; Li et al., 2016; Zhong et al., 2017; Matias et al., 2017) and non-human primate (Hayashi et al., 2015) studies. This differential responding of DA and 5-HT neurons to reward and punishment is not easy to reconcile within a simple model of two opposing neuromodulatory systems as proposed previously (Boureau and Dayan, 2011).

Other studies reveal further complexity in reward/punishment processing, specifically in the form of altered activity of non-5-HT/DA midbrain neurons. For example, DRN neurons utilising gamma-aminobutyric (GABA) were tonically inhibited during reward-waiting with further inhibition during reward acquisition, but phasically activated by aversive stimuli ( $\mathrm{Li}$ et al., 2016). In contrast, GABAergic neuronal activity in the VTA exhibited sustained activity upon rewarding cue onset but no response to the presence or absence of actual reward outcome (Cohen et al., 2012). Further, other studies found that VTA GABAergic neuronal activity was potently and phasically activated by punishment outcome, which in turn inhibited VTA DA neuronal activity (Tan et al., 2012; Eshel et al., 2015). Another study showed that glutamatergic (Glu) neurons in the DRN reinforced instrumental behaviour through VTA DA neurons (McDevitt et al., 2014).

This complexity of signalling within the DRN-VTA system in response to reward and punishment may reflect the DRN and VTA having shared afferent inputs (Watabe-Uchida et al., 2012; Ogawa et al., 2014; Dorocic et al. (2014); Beier et al., 2015; Tian et al., 2016; Watabe-Uchida et al., 2017; Ogawa and Watabe-Uchida, 2018). Another possibility is that the DRN and VTA interact with each other. Indeed, a growing number of studies have suggested that there are direct and indirect interactions among distinctive neuronal types between and within the DRN and VTA (Di Giovanni et al., 2008; Boureau and Dayan, 2011; Watabe-Uchida et al., 2012; Ogawa et al., 2014; McDevitt et al., 2014; Beier et al., 2015; De Deurwaerdère and Giovanni, 2017; Xu et al., 2017; Valencia-Torres et al., 2017; Wang, et al., 2019; Li et al., 2019). 
Taken together, information of reward and punishment signalled by neuronal activities within the DRN and VTA seems to be diverse, heterogeneous, distributed and mixed. Some of these signalling responses are illustrated in Fig. 1b. This led to the following questions (Fig. 1a). First, can these experimental findings from separate studies be reconciled and understood in terms of a single neural circuit model encompassing both the DRN and VTA? Second, can there be degenerate DRN-VTA neural circuits, which are stable? Third, can some of these degenerate circuits be identifiable, for example, through pharmacological means?

To address these questions, we developed a biologically plausible DRN-VTA computational neural circuit model based on our previous multiscale modelling framework (Joshi et al., 2017; Wong-Lin et al., 2017). The modelling takes into account known direct and indirect pathways between DRN 5-HT and VTA DA neurons, as discussed above. Upon simulating the model under reward and punishment conditions, we found that many, but not all of the experimental findings, could be captured in a single DRN-VTA model. Further, several distinct model architectures could replicate the same neural circuit activity response profile, hence demonstrating degeneracy. Applying dynamical systems theory, we found that all these circuits were dynamically stable. To distinguish the degenerated models, we simulated drug effects of DA D2-receptor based agonist and were able to distinguish between sub-groups of these seemingly degenerate model architectures. Overall, our study demonstrated the plausibility of degeneracy and stability of neural circuits of neuromodulators.

a

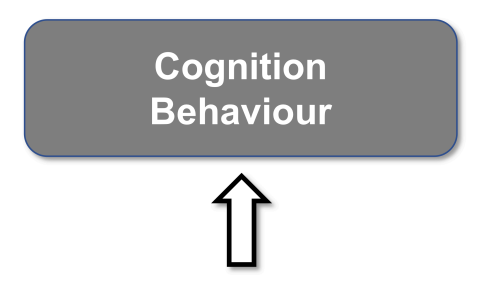

Neural circuits in other brain regions

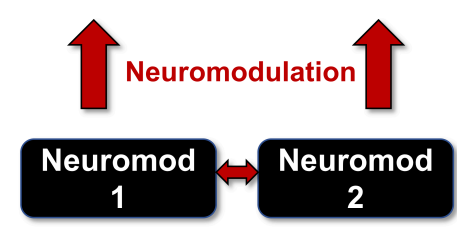

Degenerate and stable euromodulator neural circuits? b

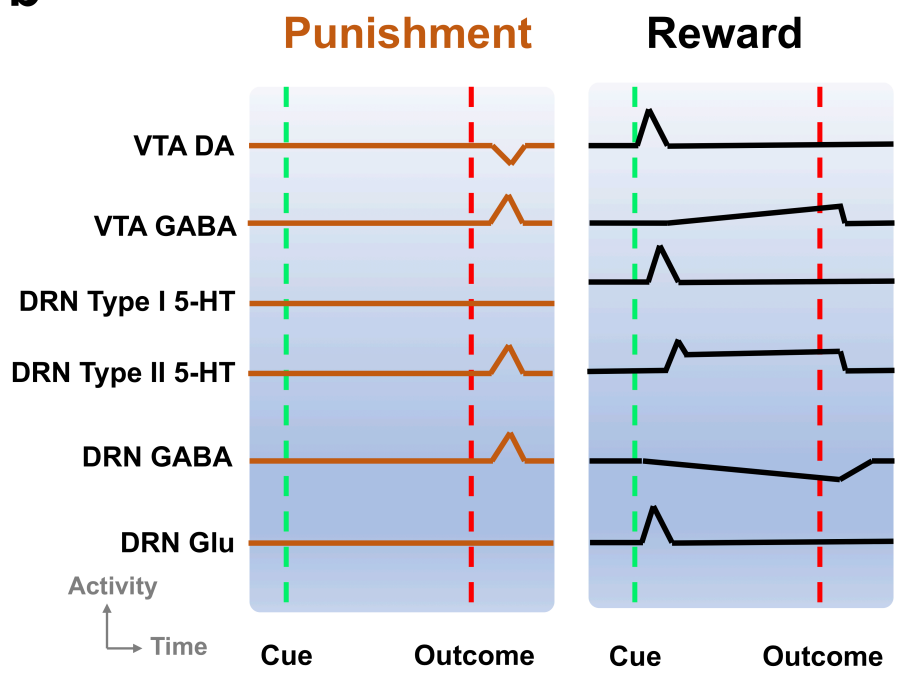

Fig. 1. Potential degenerate neuromodulator circuits constrained by stereotypical signalling. a, Multiple neuromodulators that influence neural circuits, cognition and behaviour, may be embedded within degenerate neural circuits. Neuromod: Specific neuromodulator type. $\mathbf{b}$, Schematic of DRN and VTA activity profiles in reward and punishment tasks. Activities (firing rates) aligned to timing of unexpected punishment outcome (left, vertical red dashed 
lines) and learned reward-predictive cue (right, vertical green dashed lines) and reward outcome (right, vertical red dashed lines). Top-to-bottom: VTA DA neural activity exhibits phasic excitation (inhibition) at reward-predictive cue (punishment) outcome (e.g. Cohen et al (2012), Tan et al. (2012)). VTA GABAergic neural activity shows phasic excitation upon punishment (e.g. Tan et al. (2012), Eshel et al. (2015)), while exhibiting post-cue tonic activity which is not modulated by the presence/absence of actual outcome (e.g. Cohen et al. (2012)). DRN Type-I 5-HT neurons shows phasic activation by reward-predicting cue (right) but not punishment (left). DRN Type-II 5-HT neurons signal punishment outcome (left) and sustained activity towards expected reward outcome (right) (e.g. Cohen et al. (2015)). DRN GABAergic neurons have phasic activation upon punishment but have tonic inhibition during waiting and reward delivery (e.g. Li et al. (2016)). DRN glutamatergic neurons deduced to be excited by reward-predicting cue, in line with VTA DA neural activation (McDevitt et al., 2014), and assumed not to respond to punishment outcome. Baseline activity for Type-I 5-HT DRN neurons are higher in reward than punishment tasks (e.g. Cohen et al. (2015)). 


\section{Results}

\section{Computational modelling of the DRN-VTA circuit}

To develop the DRN-VTA neural circuit models, we made use of our dynamic mean-field (neuronal population-based) modelling framework (Joshi et al., 2017) for neuromodulator interactions. The modelling approach was constrained by data from known electrophysiological, neuropharmacological and voltammetry parameters (see Methods). Each neural circuit model architecture investigated consisted of DRN 5-HT, VTA DA, VTA GABA, DRN GABA, and DRN Glu neuronal populations. Direct and indirect interactions among these five neuronal populations were then explored. The main aim of this work was to evaluate the plausibility of neuromodulator circuit degeneracy and stability rather than replicate every neuronal populations in these brain regions. Thus, DRN DA neurons were not considered in the model due to the lack of studies in standard reward/punishment conditioning tasks. VTA Glu neurons were also not considered as they constituted a lower proportion (2$5 \%$ ) of cells in this region (Yamaguchi et al., 2015).

A directed interaction between two neuronal populations, in which the source was a neuromodulator, was mathematically described by the neuromodulator neuronal population firing (rate) activity, followed by the release-and-uptake dynamics of the neuromodulator, which in turn induced certain population-averaged currents on the targeted neuronal population (Joshi et al., 2017). An induced current, $I_{x}$, which could be effectively excitatory or inhibitory depending on experimental findings, can be described by

$$
\tau_{x} \frac{d I_{x}}{d t}=-I_{x}+\frac{k_{x}}{1+e^{-g_{x}\left([x]-[x]_{0}\right)}}
$$

where $x$ was some neuromodulator (5-HT or DA), $\tau_{x}$ the associated time constant, $k_{x}$ some constant that determined the current amplitude, and constants $g_{x}$ and $[x]_{0}$ that controlled the slope and offset of the function on the right-hand-side of Equation (1). The release-and-uptake dynamics for a neuromodulator $x$ was described by

$$
\frac{d[x]}{d t}=[x]_{p} R_{x}-\frac{V_{\max , x}[x]}{K_{m, x}+[x]}
$$


where $[x]$ was the concentration of $x,[x]_{p}$ the release per neural firing frequency (Joshi et al., 2017), and constants $V_{\max , x}$ and $K_{m, x}$ were constants determined from voltammetry measurements. See Methods for further details.

If the source of the interaction came from GABAergic (or Glu) neurons, then for simplicity, we assumed an instantaneous inhibitory (or excitatory) current-based synaptic influence on the targeted neuronal populations (see Methods). This reflected the faster ionotropic receptorbased synaptic dynamics compared to metabotropic receptor-based neuromodulatory effects (Jalewa et al., 2014), while also reducing the number of free model parameters. Similarly, we also ignored the relatively faster neuronal membrane dynamics. Threshold-linear input-output function for each neuronal population was used (Jalewa et al., 2014; Joshi et al., 2017), and described by

$$
F=g\left[I-I_{0}\right]_{+}
$$

where $F$ was the neural population firing rate (output), $I$ the total input current into a neural population, $g$ was the slope, and $I_{0}$ some threshold current, and with $[I]_{+}=I$ if $I \geq 0$, and 0 otherwise. Then, $I$ is a function of the summed currents, including the neuromodulator-induced currents $I_{x}$ 's and ionotropic receptor-based currents (proportional to presynaptic neural firing rates; see Methods). For simplicity, fast co-transmission of neurotransmitters was only considered in one modelling instance (co-release of 5- $\mathrm{HT}$ and glutamate via fast $5-\mathrm{HT}_{3}$ and ionotropic receptors, in Fig. 4I) based on findings by Wang et al. (2019). From a modelling perspective, the DRN (Type I) 5-HT and Glu neuronal populations, which have rather similar activity profiles (Fig. $1 \mathrm{~b}, 3^{\text {rd }}$ and $6^{\text {th }}$ rows) could also be effectively grouped and considered as a single 5-HT neuronal population that "co-transmit" both 5-HT and Glu to DA neurons (Wang et al., 2019).

For each model circuit architecture that we investigated, we considered separately the inclusion of either Type I or II 5-HT neurons in the circuit (Cohen et al., 2015), and the possibility of excitatory and inhibitory projections from 5-HT to DRN Glu/GABA and DA neurons, and from DA to GABA neurons in the VTA (Figs. 1 and 4). To allow tractability in the search for the many possible connectivity structures, the models' connections were largely based on experimental evidence. For example, connections between VTA GABAergic and DRN Glu neurons were not considered as, to date, there is little experimental support. We also focused only on learned reward (with reward-predicting cue followed by reward outcome) and unexpected punishment conditions, simulated using a combination of tonic and/or phasic 
afferent inputs (Fig. 2; Methods). Note that we did not consider other conditions and network learning effects (e.g. Hu (2016); Zhou et al. (2018)) as the main aim was to demonstrate the plausibility of DRN-VTA circuit degeneracy and stability. This was done through the investigation of various DRN-VTA circuit architectures with network activity profiles that closely resembled those in Fig. 1b. Note also that all activity profiles in Fig. 1b were based on experimental studies except VTA Glu neural activity, which we deduced to be similar to that of DA neural activity in the reward task (McDevitt et al., 2014) and assumed to be nonresponsive in the punishment task. See Methods for further details regarding the modelling procedures, parameters, simulations, and analyses.

\section{A DRN-VTA model can reconcile many signalling patterns}

We began with a parsimonious, sparsely connected DRN-VTA model architecture and adjusted the strength of the afferent inputs and the internal connection weights of the network such that the network activity profiles attained qualitatively similar profiles to that illustrated in Fig. 1b (see Methods). The resultant network configuration (Fig. 2) was our most sparsely connected DRN-VTA circuit model. All other subsequent architecture considered would henceforth be derived from this basic architecture.

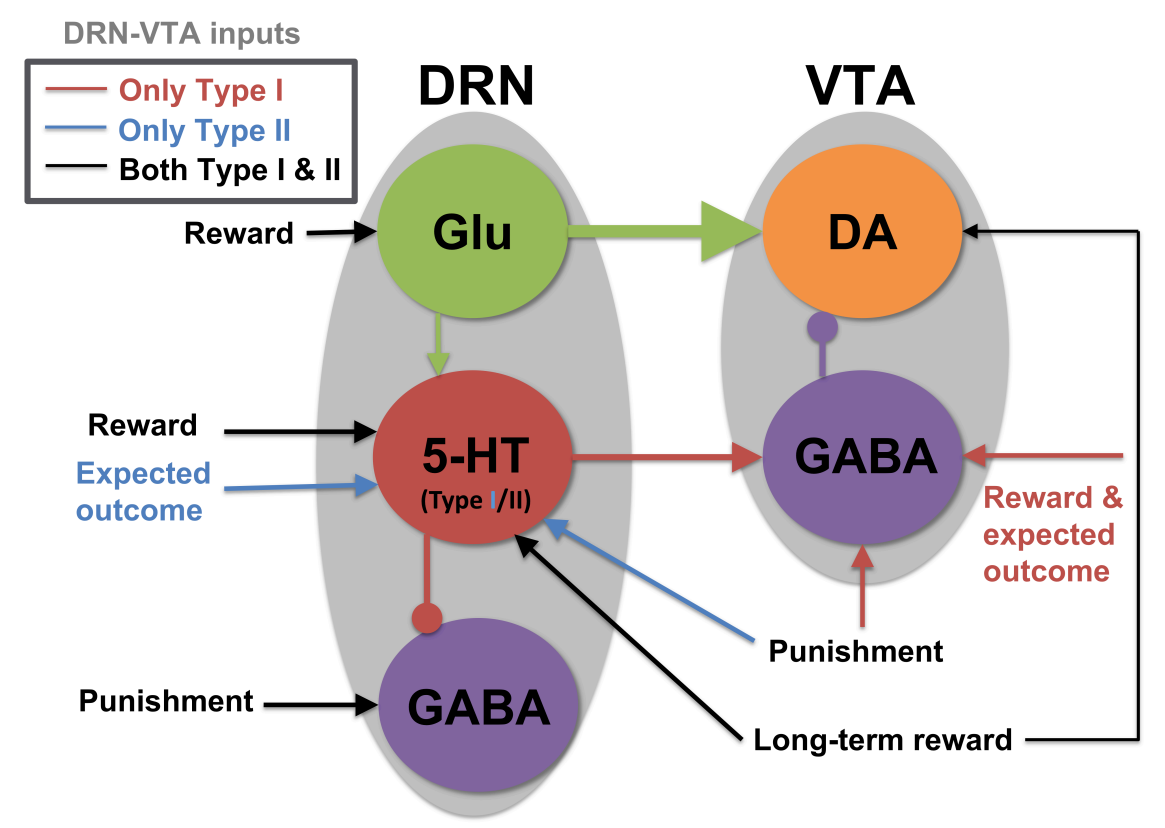

Fig. 2. A sparsely connected DRN-VTA circuit model. Grey: brain region. Coloured circle: neuronal population. Legend: network's afferent inputs. Model architecture implicitly encompasses either Type I or II 5-HT neurons with two different inputs for reward/punishment task (bright red arrows if Type I; blue arrows if Type II; black arrows denote common inputs for reward/punishment task for both Types). Circuit connections: triangular-end arrows (excitatory); circle-end arrows (inhibitory). Thicker arrows: stronger connection weights. 
Constant long-term reward inputs simultaneously to 5-HT and DA neurons to alter baseline activities. Sustained activity for expectation of reward outcome implemented with tonic input between cue and reward outcome. All other inputs are brief, at cue or reward/punishment outcome, producing phasic excitations/inhibitions. Note: Self-inhibitory (self-excitatory) connections within GABAergic (Glu) neurons, and autoreceptor inhibitions within 5-HT or DA neurons were implemented (see Methods, and Supplementary Fig. 1) but not shown here. This is the most sparsely connected model architecture considered in this work.

This minimal network architecture readily recapitulated many of the neuronal signalling changes in the DRN and VTA in separate experimental studies, both for reward (Fig. 3, black dashed) and punishment (Fig. 3, orange bold) tasks. Moreover, only modifications to afferent inputs to DRN 5-HT and VTA GABA neurons (see Methods) were required to replicate the signalling of Type I or II 5-HT neurons (Figs. 3a and b, respectively), while maintaining the same internal connectivity structure. For example, a lack of sustained reward-based activity of Type I 5-HT activity (Fig. 3b, $2^{\text {nd }}$ row, black dashed) required additional external input to sustain VTA GABAergic neural activity (Fig. 2; Fig. 3a, bottom row, black dashed). It should be noted that this was based on the assumption that the activity profiles for these non-5-HT neurons were qualitatively similar, regardless of the 5-HT neuronal types (Fig. 2). See Supplementary Notes 1 for detailed discussion on modelling the activity profiles.
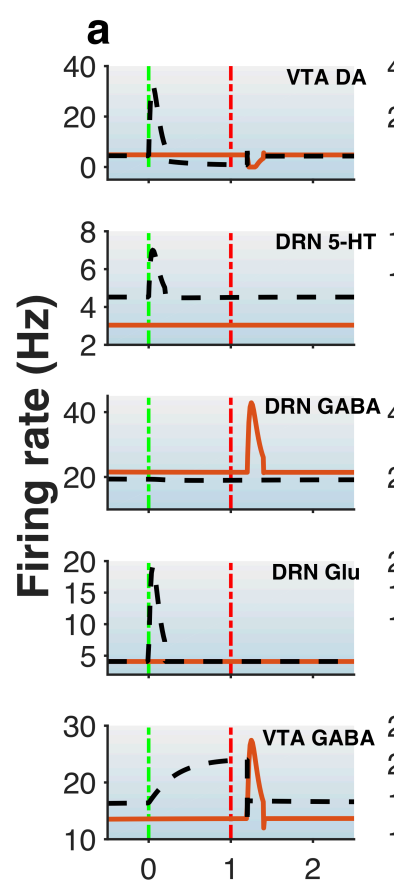

Time (s)
C

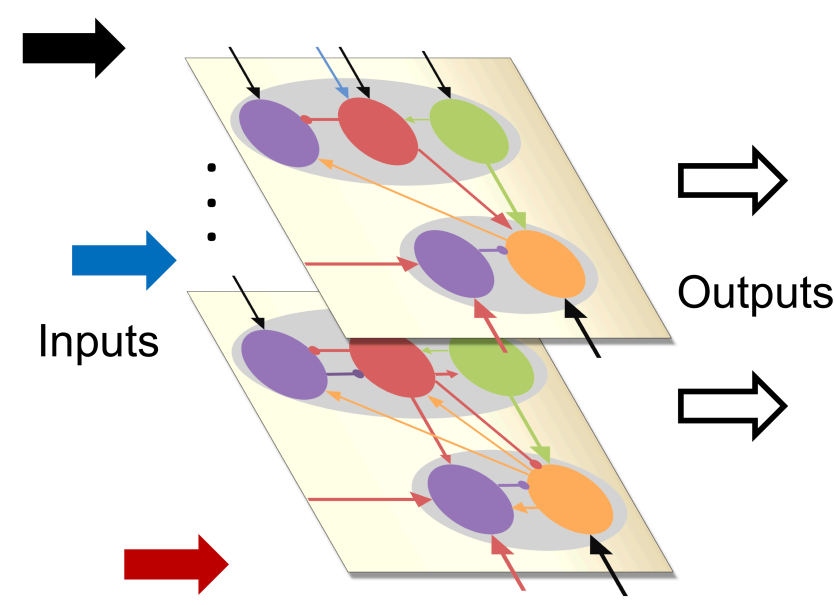

Fig. 3. DRN-VTA model replicates signalling patterns and suggests multiple parallel circuits. a-b, Model with reward (black dashed lines) and punishment (orange bold lines) tasks with 5-HT neurons that are of Type I (a) or Type II (b). Time label from cue onset. Green (red) vertical dashed-dotted lines: cue (outcome) onset time (as in Fig. 1b). Top-to-bottom: 
VTA DA, DRN 5-HT, DRN GABAergic, DRN Glu, and VTA GABAergic neural populations. c, Hypothesis for multiple different DRN-VTA circuits operating in parallel, which may consist of different clusters of neuronal sub-populations and different set of afferent inputs. Vertical dots denote the potential of having more than two distinctive circuits.

To understand across-trial reward versus punishment effects in the model, we implemented a higher constant excitatory input into both DRN 5-HT and VTA DA neurons under reward compared to punishment conditions (Fig. 2, long black arrows). This particular model required differential inputs to 5-HT and DA neurons (Methods) such that the overall tonic 5-HT neural activity was higher for reward than punishment trials, while DA neural activity remained unchanged (Fig. 4, black dashed vs orange bold lines in top two rows), again consistent with experimental observation (e.g. Cohen et al., 2015). In the model, although both 5-HT and DA neurons directly received constant across-trial reward-based excitatory inputs, the indirect inhibitory pathway from 5-HT neurons through VTA GABA neurons onto VTA DA neurons nullified the overall effects on DA neurons (Figs. 2 and 3). In other words, increased firing of 5-HT neurons could be activating VTA GABAergic neurons to a level sufficient to inhibit VTA DA neurons and thereby cancelling out the net long-term reward signals (Fig. 2). See Supplementary Notes 2 for discussion on the effects and implications of phasic DRN Glu neuronal activity.

However, under these conditions, with both Types I and II 5-HT neurons, the baseline DRN and VTA GABAergic activities in the reward task were slightly different than those in the punishment task (Fig. $3,3^{\text {rd }}$ and $5^{\text {th }}$ rows, black dashed vs orange bold), which have yet to be observed in experiments. The model's inability to recapitulate all experimental findings with a single neural circuit architecture might perhaps suggest that there are more complex features in the system, such as further division of neuronal subgroups. This also holds for other model architectures (see below, Fig. 4). For example, a sub-population of DRN GABAergic neurons might be directly connected to 5-HT neurons (as in Fig. 1b), while another DRN GABAergic neuronal sub-population might not be connected such that across-trial reward signal inputs are distributed differently than that of Fig. 1b. Moreover, high chemical and functional diversity amongst DRN 5-HT neurons is now well recognised (Okaty et al., 2019). Hence, it might be possible that there could exist multiple neural circuits with different circuit architectures operating in parallel, as illustrated in Fig. 3c.

Taken together, we have shown that, under reward and punishment conditions, many of the observed signalling patterns in different DRN and VTA neuronal types could readily be 
reconciled within a single sparsely connected DRN-VTA circuit model. However, not all the signalling patterns could be captured, suggesting that multiple different neuronal subpopulations and circuits may be operating in parallel within the DRN-VTA system. Next, we shall investigate whether various different DRN-VTA neural circuits could produce the same output, i.e. be degenerate.

\section{Multiple degenerate DRN-VTA circuits}

To search for degenerate DRN-VTA models, we evaluated various combinations of connections within and between the DRN and VTA, and where necessary, adjusted any afferent inputs (Methods). We used the network activity profile illustrated in Figs. 3a and $b$ as an output "target" to check whether other different circuits could replicate a similar activity profile. Given the variability of neuronal firing rates reported in the literature, to be considered a degenerate neural circuit, we set an inclusion criterion that permitted maximal deviations of the firing activities of the DRN 5-HT, DRN GABA, DRN Glu, VTA DA, and VTA GABA neural populations to be less than $10 \%, 17 \%, 10 \%, 10 \%$ and $17 \%$, respectively, from those illustrated in Fig. 3, respectively (see Methods, Supplementary Table 1, and Supplementary Fig. 2). This was quantified within a $3 \mathrm{~s}$ time duration, from $1 \mathrm{~s}$ before cue onset to $1 \mathrm{~s}$ after outcome onset, encompassing both baseline and stimulus-evoked activities. Within our inclusion criteria, we discarded any neural circuit architecture which could not replicate the activity profiles shown in Fig. 3.

Various neural circuits were created by systematic addition and modification of connections of our sparsely connected DRN-VTA circuit (now presented as Fig. 4k), until we reached a highly connectivity model structure (Fig. 4a) yet which was constrained by experimental findings (see above). Each of the circuits was simulated and evaluated in both reward and punishment tasks using both Type I and II 5-HT neurons, as done above. Both excitatory and inhibitory connections from DRN 5-HT to DRN Glu/GABA and VTA DA neurons were explored. Thus, based on these combinations, we obtained a total of 84 different neural circuit model architectures (including the one in Fig. 3 and its variants, as Fig. 4k) that fitted our definition of degeneracy (with respect to Fig. 3). The high-level model architectures were illustrated in Fig. 4 (see also Fig. 5, and Supplementary Tables 2 and 3). For instance, model architecture 'a' in Fig. 4a (see Supplementary Fig. 1 for detailed architecture) actually consisted of 32 distinctive models with different 5-HT neuron types and connectivity signs (see Supplementary Table 3). Interestingly, the model parameters remained the same with either excitatory or inhibitory connections (Fig. 2, diamond connections; Supplementary Table 2). Supplementary 
Fig. 2 shows an example of the percentage change in firing rates (from activity profile template) with model architecture 'l' and an excitatory 5-HT-to-DA connection, using Type-I 5HT neurons under the reward task. See Supplementary Notes 3 for further discussion on the connectivity of the degenerate models and how they address mixed findings in the literature.

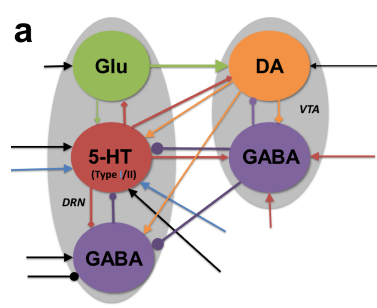

e
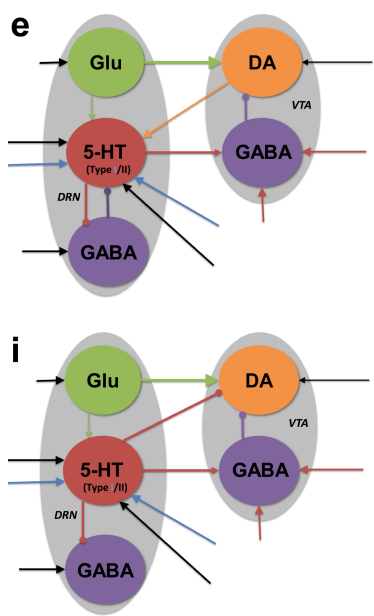
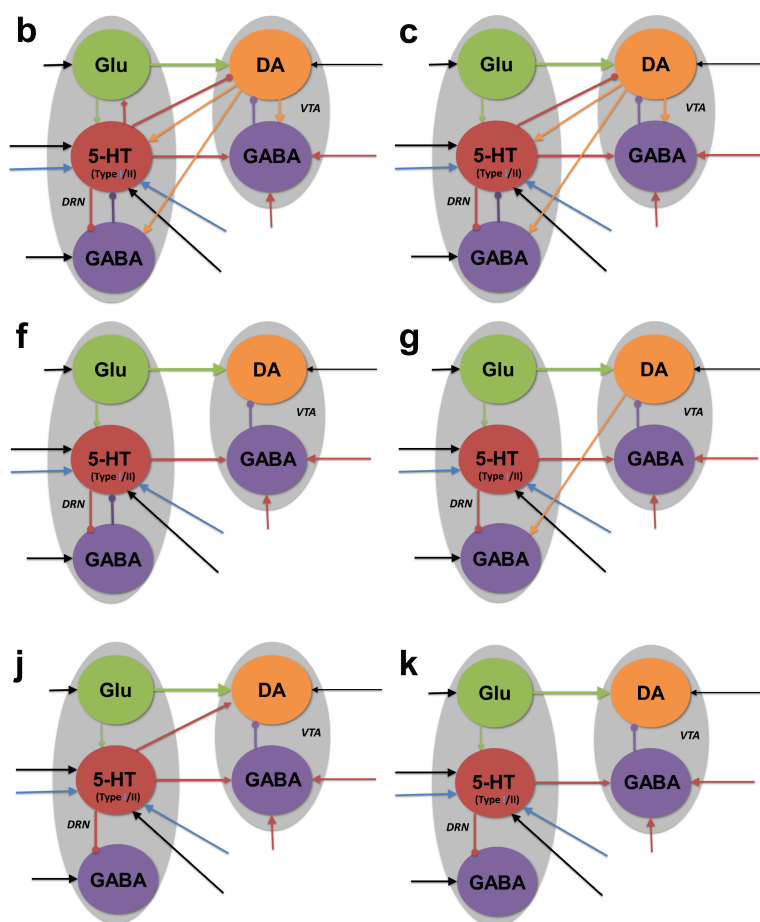

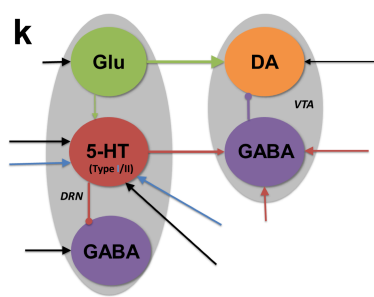

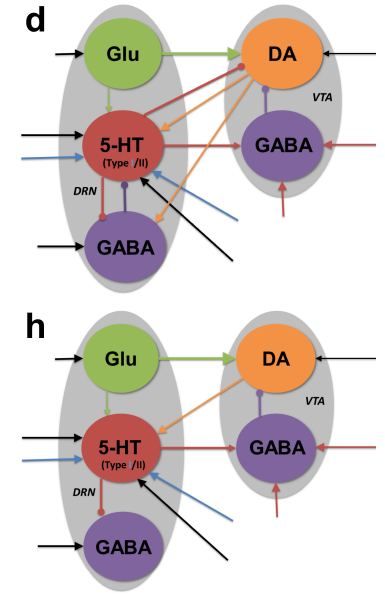

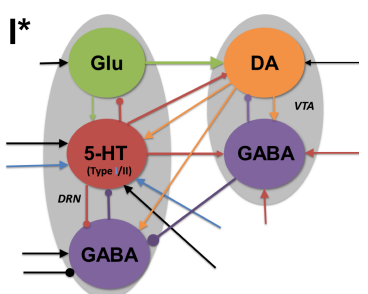

Fig. 4. Neural circuit model architectures with similar network activity profiles. The activity profiles (not shown) were similar to that in Figs. 3a and b, confining within set ranges of values (see text). a-k, Architectures of decreasing connectivity, with Fig. 3 in (I). I, with an asterisk denotes the only model with fast 5-HT to VTA DA connection, simulating fast 5- $\mathrm{HT}_{3}$ or Glu receptor mediated connection or their combination (co-transmission). a, I, Additional inhibitory input to DRN GABA neurons in reward task. All labels, connections and nomenclature have the same meaning as that in Fig. 2, except that, for simplicity, the relative connection weights (thickness) are not shown, and the diamond-end arrows denote connections which are either excitatory or inhibitory, with both explored. Self-connectivity not shown (see an example in Supplementary Fig. 1 for a detailed version of Fig. 4a). Note: Each architecture consists of several distinctive model types (with a total of 84 types) with different 5-HT neuronal or excitatory/inhibitory connectivity types (see Supplementary Tables 2 and 3, and Fig. 5).

\section{Degenerate DRN-VTA circuit models are dynamically stable}

After identifying the above degenerate models, we used dynamical systems theory to determine whether they were dynamically stable, i.e. whether (local) perturbation from their steady states would eventually cause a return to their initial steady states (see Methods). Specifically, the stability of each neural circuit could be determined by first finding the possible steady state(s) (i.e. fixed point(s)). This was achieved by setting all the dynamical (differential) 
equations to zero and finding the algebraic solutions for the dynamical variables (Eqns. 1-2; Methods). Then the eigenvalues of the system's Jacobian matrix at the steady states were computed (see Methods for mathematical derivation of the steady states and the Jacobian matrix). For a neural circuit to be dynamically stable, the real part of all the eigenvalues associated with the steady state has to be negative. This was exactly what we found for all the degenerate neural circuits in Fig. 4 (Fig. 5; Methods), with no imaginary part in the eigenvalues.

Fig. 5a plotted the complete set of the real part of the eigenvalues for model \#1 with architecture 'a' (Fig. 4a). This model has inhibitory connections from VTA DA to VTA GABA neurons and from DRN 5-HT to VTA DA/Glu neurons, using Type I 5-HT neurons and under punishment conditions (Supplementary Table 3). It was observed that the eigenvalues with a phasic input (blue) were generally larger (magnitude wise) than those with tonic input (red). This was more pronounced for the eigenvalues with the largest magnitude (maximal eigenvalues) (Fig. 4a, asterisk). Moreover, all the eigenvalues were negative, indicating a dynamically stable network model even in the presence of additional phasic stimulus input. The non-maximal eigenvalues were similar to those of the other models (not shown).

We repeated the analysis for all 84 models, under both phasic and tonic input conditions. This analysis is presented in Fig. 5b only for the maximal eigenvalues (red circles and blue crosses). Note that for each model, different 5-HT neuron, connectivity types (excitatory/inhibitory), and tasks, were evaluated (e.g. model 'a' had 32 different types) (Supplementary Tables 2 and 3). In general, with phasic activities (blue crosses), the models were more stable than with tonic activities (red circles). However, during phasic activations, there were 18 models with rather small (close to zero) eigenvalues (magnitude wise), albeit still negative. This was not observed for tonic activations, where the (most negative) eigenvalues were found to hover within a small range of values $(-0.017$ to -0.016$)$, except models with architecture ' $l$ ' ( -0.03). In fact, the latter models, which were the only ones with a fast 5-HT-to-DA connection (Fig. 5b, models \#77-84), were the most stable under both phasic and tonic conditions. The other non-maximal eigenvalues remain similar to those of the other models (not shown). Further, there was no difference identified between the excitatory (models \#77-80) and inhibitory (models \#81-84) connections. Moreover, most of their eigenvalues in phasic condition were more negative than their tonic counterparts. 
a

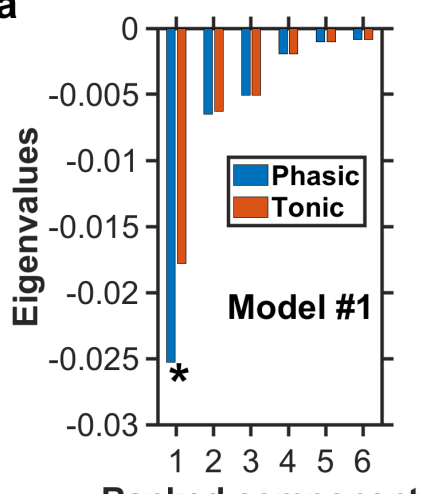

b

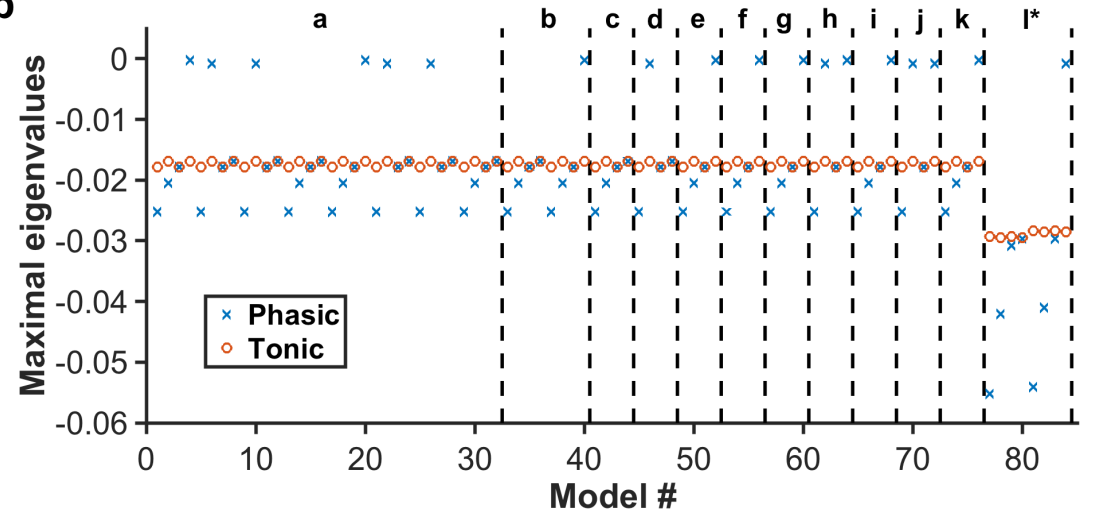

Fig. 5. Negative real eigenvalues at steady states of degenerate models. a, Complete set of the real part of the eigenvalues for Model \#1 (Supplementary Tables 2 and 3) with architecture illustrated in Fig. 4a. Horizontal axis: Components from PCA ranked from the largest to the smallest eigenvalues (magnitude wise). Blue (red): More negative eigenvalues with phasic (blue) than tonic (red) input. Asterisk: Leading/Maximal eigenvalue (largest magnitude) for each input condition. b, For each of the 84 models, only the real part of the eigenvalue with the largest magnitude is plotted under phasic (blue cross) and tonic (red circle) input condition. Model category a to I* refer to the different architectures in Fig. 4, in which each has their own distinctive model types (e.g. different 5-HT neuronal or excitatory/inhibitory connectivity types). Eigenvalues for all model types have negative real parts, indicating dynamically stable. For most models, the eigenvalues are generally more negative during phasic than tonic activities.

\section{$D_{2}$ mediated drugs can distinguish some degenerate DRN-VTA circuits}

Given the large number of degenerate and stable DRN-VTA circuits that were possible, how can one distinguish among at least some of them? To address this, we investigated the circuit responses to simulated $D_{2}$ receptor agonists. This approach was selected due to the extensive $D_{2}$ receptor mediated connectivity within the degenerate DRN-VTA circuits (Fig. 4). In particular, these connections involved those from VTA DA neurons to DRN 5-HT, DRN GABA and VTA GABA neurons, and also self-inhibition ( $D_{2}$ autoreceptor-mediated) of DA neurons (see Methods for references). To mimic the effects in the model of $D_{2}$ receptor agonist drugs, we gradually increased the strengths of connections mediated by $D_{2}$ receptors (Supplementary Fig. 1, orange connections emanating from DA neurons) by some factor (X) and observed the neural activity changes.

As we gradually increased the strengths of these specific sets of connection, subsets of the degenerate models gradually behave differently from the activity profile template of Figs. $3 a$ and $b$ (Figs. 6a and b), allowing us to distinguish between these model groups. Fig. $6 c$ showed an example of such differences. However, such evaluation under punishment condition (Fig. 6a) was slightly more limited than reward condition (Fig. 6b) in terms of distinguishing the 
degenerate circuits. See Supplementary Notes 4 for detailed discussion at each discrete increment of connection strength. Interestingly, these effects of $D_{2}$ receptor activation remained regardless of whether the circuits encompassed Type I or Type II 5-HT neurons. Thus, the modelling predicted that a gradual increment of the level of $D_{2}$ receptor activation (such as through administration of $D_{2}$ agonist drugs) could lead to differential suppressions and/or enhancements of firing rate activities that could be used to distinguish subsets of the degenerate DRN-VTA circuits.

a

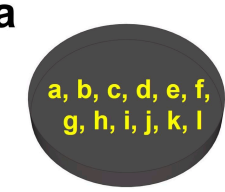

$x=1$

b

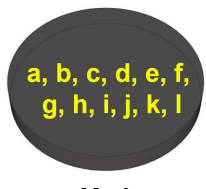

$x=1$

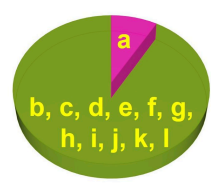

$x=10$

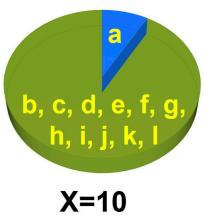

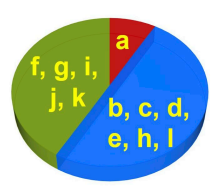

$X=40,70$

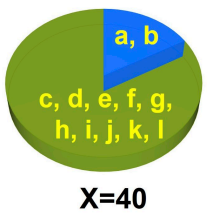

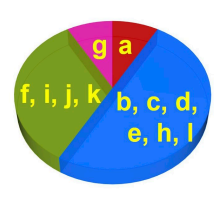

$x=100$

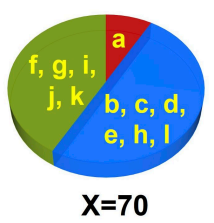

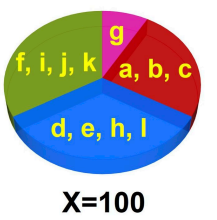

$x=100$

Control DA $\uparrow$

DA $\uparrow \& 5-H T \uparrow$

DA $\uparrow \&$ GABA (DRN) $\uparrow$

DA $\uparrow$, 5-HT $\uparrow \&$ GABA (DRN)
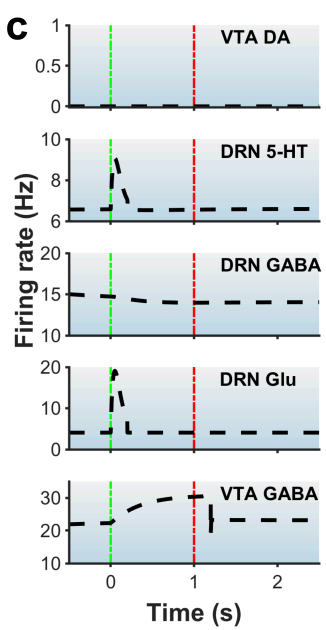

Fig. 6. $D_{2}$ receptor agonist can distinguish subsets of DRN-VTA neural circuits. $a-b$, Drug administered in punishment (a) and reward (b) task with efficacy factor $X$ increments of $1,2,10,40,70$ and 100 times. Letters in yellow label model circuit architectures as in Fig. 4. (See Supplementary Tables 4-8 for details.) c, High $D_{2}$ receptor agonist substantially changed DA and 5-HT activities with model architecture 'e' and Type-I 5-HT neurons in reward task. $\mathrm{D}_{2}$ agonist dosage was enhanced by 100 times (compared with Fig. 3). Time label from cue onset.

\section{Discussion}

In this work, we addressed whether neural circuits that utilised neuromodulators can themselves be degenerate by computationally modelling DRN-VTA circuits, which shared structural and functional structural and functional bidirectional relationship among their constituent neuron types. Moreover, these circuits are involved in the regulation of key cognitive, emotional and behavioral processes, and implicated in many common and disabling neuropsychiatric conditions. To begin, we developed a biologically-based, mean-field computational model of the DRN-VTA circuit (Fig. 2) with several neuronal types, and tested it under classic conditions of reward and punishment. The modelling was partially constrained by known connectivity within and between the DRN and VTA regions, and their inputs from 
multiple other brain regions, including mixed combinations of inputs (Watabe-Uchida et al., 2012; Ogawa et al., 2014; Dorocic et al. (2014); Beier et al., 2015; Tian et al., 2016; WatabeUchida et al., 2017; Ogawa and Watabe-Uchida, 2018).

We found that a parsimonious, sparsely connected version of the DRN-VTA model could reconcile many of the diverse phasic and tonic neural signalling events reported in the DRN and VTA in (unexpected) punishment and (learned) reward tasks observed across separate experimental studies (Figs. 1 and 3). This model was evaluated using Type I and Type II 5HT neurons in the DRN as defined electrophysiologically in a previous study (Cohen et al., 2015). In the case of Type II 5-HT neurons under the reward task, the model predicted that sustained 5-HT neuron activity between cue and reward outcome (Cohen et al., 2015) would lead to the gradual inhibition of DRN GABA neuron activity and enhancement of VTA GABA neuron activity, as previously observed experimentally (Cohen et al., 2012; Li et al., 2016). The sparsely connected model could also reproduce experimental observations (Cohen et al., 2015) of an increase in baseline firing of Type I 5-HT neurons across several trials in the rewarding task, without similar effects on VTA DA neurons, or in the punishment task (Fig. 3). This model suggested that slow, across-trial reward-based excitatory inputs could potentially be directly targeted to both DRN 5-HT and VTA DA neurons, and that inhibitory 5-HT to GABA to DA connectivity cancelled out the effects of the direct input to VTA DA neurons, rendering only long timescale changes on baseline activity of DRN 5-HT neurons (Fig. 2). Such a cancellation effect is reminiscent of parallel excitatory and inhibitory pathways operating in conditioning tasks (e.g. Zhou et al. (2018)).

Despite the strong predictive validity of the model, it was not able to fully capture some of the activity profiles with Type I 5-HT neurons, namely, the differential baseline activities of VTA GABA neurons and DRN GABA neurons between reward and punishment conditions. Perhaps our model of DRN-VTA circuit might not be sufficiently complex to capture all the signalling effects reported in experimental studies, even though we observed similar results with more complex model architectures (e.g. as in Figs. 4a and I). Thus, further additional neuronal populations and DRN-VTA circuits could be operating in parallel (Fig. 3c). Such parallel circuits could be validated experimentally in the future, for example, using genetargeting of specific DRN and VTA neuron subtypes and projections. Indeed, there is increasing evidences that DRN 5-HT neurons are more chemically diverse than previously expected, and that there is a high level of functional diversity in output pathways of the DRN and VTA (Watabe-Uchida et al., 2012; Ogawa et al., 2014; Dorocic et al. 2014; Weissbourd et al., 2014; Beier et al., 2015; Tian et al., 2016; Fernandez et al., 2016; Watabe-Uchida et al., 
2017; Zhou et al., 2017; Morales and Margolis, 2017; Ren et al., 2018; Ogawa and WatabeUchida, 2018; Okaty et al., 2019).

To demonstrate degeneracy in the DRN-VTA system, we showed that several variants of the DRN-VTA circuit model could readily recapitulate the same neural signalling profiles, even with occasional slight changes made to their afferent inputs (Fig. 4). We then applied dynamical systems theory and showed that all these degenerate circuits were dynamically stable (Fig. 5). Compared to the sparsely connected model, more highly connected and degenerate versions of the DRN-VTA model predicted a relatively weaker direct DRN 5-HTVTA DA neuron connectivity than that of DRN Glu-VTA DA neurons (McKevitt et al., 2014). However, previous studies had demonstrated a direct influence of DRN 5-HT on VTA DA neuron activity as well as reward (e.g. De Deurwaerdère and Di Giovanni (2017)). More recent work has shown that DRN 5-HT terminals in the VTA co-release glutamate and 5-HT, eliciting fast excitation (via ionotropic receptors) onto VTA DA neurons and increased DA release in the nucleus accumbens to facilitate reward (Wang et al., 2019). Hence, we developed a model of this fast 5-HT-to-DA connection and found such system to be plausible in terms of capturing the stereotypical reward and punishment signalling (Fig. 4I). Interestingly, we found that this model configuration was dynamically more stable than all other architectures (Fig. 5b). Future modelling work could explore the effects of co-transmission of neurotransmitters on neural circuit degeneracy and functioning. This may require the involvement of more biologically realistic spiking neuronal network models across multiple scales (Cullen and Wong-Lin, 2015; Canavier et al., 2016; Wong-Lin et al., 2017).

Finally, we simulated increased $D_{2}$ receptor activation by increasing the connection strengths emanating from the VTA DA neurons. This allowed us to distinguish some of the degenerate DRN-VTA neural circuits by identifying substantial deviations in specific neural population activities (Figs. 6a and b). Interestingly, more degenerate neural circuits could be identified in rewarding than in punishment tasks (compare Fig. 6a to Fig. 6b). Experimental verification of such results could pose a challenge. Together, our computational modelling and analytical work supported the existence of degeneracy and stability in the DRN-VTA circuits. Some of these degenerate circuits were dynamically more stable than others, and a subset of the degenerate circuits could be distinguished through pharmacological means.

From a more general perspective, our computational modelling and analytical framework could be applied to the study of degeneracy and stability of neural circuits involving the interactions of other neuromodulators such as norepinephrine/noradrenaline (e.g. Jalewa et al., 2014; Joshi et al., 2017). It should also be noted that in the development of the models, 
we had resorted to a minimalist approach by focusing only on sufficiently simple neural circuit architectures that could replicate closely to the experimentally observed data. Future modelling work may investigate the relative relevance of these connections with respect to larger circuits involving cortical and subcortical brain regions across multiple scales, especially during adaptive learning (e.g. Wang and Wong-Lin, 2013; Zhou et al., 2018).

Overall, through computational modelling and mathematical analysis, our study suggests the plausibility of degenerate and stable neural circuits that encompass serotonin and dopamine neuromodulators. More generally, the work opens up a new avenue of investigation on degeneracy in neuromodulatory circuits and has important implication on the stable and robust maintenance of neuromodulatory functions.

\section{Methods}

\section{Input-output functions of neural population firing rates}

The computational models developed were based on our previous mean-field, neural population based modelling framework for neuromodulator circuits (Joshi et al., 2017), in which the averaged concentration releases of neuromodulators (e.g. [5-HT]) were monotonic functions of the averaged firing rate of (e.g. 5-HT) neuronal populations via some neuromodulator induced slow currents. All 5 neural populations' firing rates were described by threshold-linear functions (general form in Eqn. (3)) (Jalewa et al., 2014; Joshi et al. 2017):

$$
\begin{gathered}
F_{5-H T}=g_{5-H T}\left[I_{5-H T}-I_{0,5-H T}\right]_{+} \\
F_{D A}=g_{D A}\left[I_{D A}-I_{0, D A}\right]_{+} \\
F_{G l u}=g_{G l u}\left[I_{G l u}-I_{0, G l u}\right]_{+} \\
F_{G A B A-D R N}=g_{G A B A-D R N}\left[I_{G A B A-D R N}-I_{0, G A B A-D R N}\right]_{+} \\
F_{G A B A-V T A}=g_{G A B A-V T A}\left[I_{G A B A-V T A}-I_{0, G A B A-V T A}\right]_{+}
\end{gathered}
$$

where $[x]_{+}=x$ if $x \geq 0$, and 0 otherwise. The threshold input values for $I_{0, D A}$ was -10 (a.u.) for DA neurons, and $I_{0,5-H T}$ was 0.13 (a.u.) for 5-HT neurons, to allow spontaneous activities mimicking in vivo conditions (Fig. 3). 5- $\mathrm{HT}$ neurons had a threshold-linear function with gain value $g_{5-H T}$ of about 1.7 times higher than that for DA neurons, and so we set their for DA and 5-HT neurons to be 0.019 and $0.033(\mathrm{~Hz})$, respectively (e.g. Shepard and Bunney, 1991; Richards et al., 1997; Crawford et al., 2010; Wong-Lin et al., 2012; Challis et al., 2013). For simplicity, we assumed the same current-frequency or input-output function in either tonic or phasic activity mode (Jalewa et al., 2014; Joshi et al., 2017). 


\section{Afferent currents and connectivity}

The afferent current, $I$, for a neural population consisted of summed contributions from external excitatory inputs $I_{\text {ext }}$ including those induced by reward or aversive stimuli, and recurrent interactions with other neural populations (see below) e.g. $I_{5-H T, D A}$ for effective DAinduced currents in 5-HT neurons. Additionally, for a neuromodulator population, autoreceptor-induced current, $I_{\text {auto }}$, was included.

Due to limited experimental evidences, and to reduce the parameter search space, we did not consider the following connections from: (i) DRN GABA to VTA DA neurons; (ii) DRN GABA to VTA GABA neurons; (iii) VTA GABA to DRN Glu neurons; (iv, v) DRN Glu to DRN GABA neurons, and vice versa; and (vi) VTA DA to DRN Glu neurons. Then the total (populationaveraged) afferent input currents to DA and 5-HT neurons were, respectively, described by

$$
\begin{gathered}
I_{D A}=-I_{D A, \text { auto }} \pm I_{D A, 5-H T}+I_{D A, G l u}-I_{D A, G A B A-V T A}+I_{D A, \text { ext }} \\
I_{5-H T}=-I_{5-H T, \text { auto }}+I_{5-H T, D A}+I_{5-H T, G l u}-I_{5-H T, G A B A-D R N}-I_{5-H T, G A B A-V T A}+I_{5-H T, \text { ext }}
\end{gathered}
$$

where the first terms on the right-hand sides of Eqns. (6) and (7) were autoreceptor-induced self-inhibitory currents, the second terms were the 5-HT-to-DA (labelled with subscript $D A, 5-$ $H T$ ) and DA-to-5-HT (with subscript $5-H T, D A$ ) interactions, the third terms were excitatory interactions from DRN Glu neurons, the fourth/fifth terms were inhibitory interactions from local DRN/VTA GABAergic neurons, and the last terms were additional external constant biased inputs from the rest of the brain and the influence of behaviourally relevant stimuli (due to rewards or punishments; see below). 5-HT neurons have a possible additional negative interaction from VTA GABA neurons (second last term on right-hand-side) (Li et al., 2019). Negative or positive sign in front of each term indicated whether the interaction was effectively inhibitory or excitatory. The \pm sign indicated effectively excitatory $(+)$ or inhibitory $(-)$ interactions which we investigated (Figs. 2, 4 and 5), given their mixed findings in the literature (see also Eqns. (11-13)). This form of summed currents was consistent with some experimental evidence that showed different afferents modulating the tonic and phasic activation (e.g. Floresco et al. (2003); Tian et al. (2016)).

Similarly, the total (population-averaged) afferent current to the glutamatergic (Glu), and VTA and DRN GABAergic neurons, can respectively be described by

$$
\begin{gathered}
I_{G l u}=I_{\text {self }, G l u} \pm I_{G l u, 5-H T}+I_{G l u, \text { ext }} \\
I_{G A B A-D R N}=-I_{\text {self }, G A B A-D R N} \pm I_{G A B A-D R N, 5-H T} \\
+I_{G A B A-D R N, D A}-I_{G A B A-D R N, G A B A-V T A}+I_{G A B A-D R N, \text { ext }} \\
I_{G A B A-V T A}=-I_{\text {Self }, G A B A-V T A}+I_{G A B A-V T A, 5-H T} \pm I_{G A B A-V T A, D A}+I_{G A B A-V T A, \text { ext }}
\end{gathered}
$$


where, the subscript Glu denoted DRN Glu neural population, and subscripts $G A B A-V T A$ and $G A B A-D R N$ denoted GABAergic neural populations in the VTA and DRN, respectively. The subscript self denoted self-connection.

The averaged synaptic currents of non-5-HT/DA ionotropic glutamatergic \& GABAergic neurons, namely, $I_{D A, G l u}, I_{5-H T, G l u}, I_{D A, G A B A-V T A}, I_{5-H T, G A B A-V T A}, I_{5-H T, G A B A-D R N}, I_{\text {self,Glu}}$, $I_{\text {self,GABA-DRN }}, I_{\text {self,GABA-VTA }}$ and $I_{G A B A-D R N, G A B A-V T A}$ were typically faster than currents induced by (metabotropic) 5-HT or DA currents. Thus, we assumed the former currents to reach quasi-steady states and described (Jalewa et al., 2014) and represented by $I_{e / i}=$ $\pm J_{e / i} F_{e / i}$, where the subscript $e / i$ denoted excitatory/inhibitory synaptic current, $J$ the connectivity coupling strength (Methods), $F$ the presynaptic firing rate for neural population $e / i$, and the sign \pm for excitatory or inhibitory currents. Further, dimensionless coefficients or relative connectivity weights, $W^{\prime} s$ (with values $\geq 0$ ), were later multiplied to the above neuromodulator induced current terms (right-hand-side of terms in Eqs. (9-13); see Eqns. (20$24)$ ). Both the $J^{\prime} s$ and $W^{\prime} s$ were allowed to vary to fit the network activity profiles of Fig. $1 \mathrm{~b}$ within certain tolerance ranges (see below) while exploring different neural circuit architectures (Fig. 4) (see Supplementary Table 2 for specific values). The self-connection weights $J$ 's within the DRN Glu, DRN GABA and VTA GABA neurons were set at $0.5,0.5$, and 10 respectively, for all network activity's response profiles.

Autoreceptor-induced currents were known to trigger relatively slow $G$ protein-coupled inwardly-rectifying potassium (GIRK) currents (Tuckwell and Penington, 2014). For 5-HT $1 \mathrm{~A}$ autoreceptors, the inhibitory current $I_{5-H T \text {,auto }}$ was described by (Ritter et al., 2008; Tuckwell and Pennington, 2014; Joshi et al., 2017)

$$
\tau_{\text {auto }, 5-H T} \frac{d I_{\text {auto }, 5-H T}}{d t}=-I_{\text {auto }, 5-H T}+\frac{k_{\text {auto, } 5-H T}}{1+e^{-g_{a u t o, 5-H T}([5-H T]-[5-H T] 0)}}
$$

and similarly, for DA autoreceptor induced inhibitory current $I_{\text {auto,DA }}$ :

$$
\tau_{\text {auto }, D A} \frac{d I_{\text {auto }, D A}}{d t}=-I_{\text {auto }, D A}+\frac{k_{\text {auto }, D A}}{1+e^{-g_{\text {auto }, D A}\left([D A]-[D A]_{0}\right)}}
$$

where $\tau_{\text {auto, } 5-H T}$ was set at $500 \mathrm{~ms}$ (Joshi et al., 2017) and $\tau_{\text {auto,DA}}$ at $150 \mathrm{~ms}$ (Benoit-Marand et al., 2001; Courtney et al., 2012; Cullen and Wong-Lin, 2015). The threshold values [5 $H T]_{0}$ and $[D A]_{0}$ were set at $0.1 \mu \mathrm{M}$. These parameters can be varied to mimic the effects of autoreceptor antagonists/agonist (Joshi et al., 2017). The gains $g_{a u t o, 5-H T}$ and $g_{a u t o, D A}$ were set at $10 \mu \mathrm{M}^{-1}$ each, and $k_{\text {auto, } 5-H T}=k_{\text {auto,DA }}=80$ a.u.. These values were selected to allow reasonable spontaneous neural firing activities and baseline neuromodulator concentration levels (see below), and similar to those observed in experiments.

Similarly, we assumed sigmoid-like influence of $[5-H T]([D A])$ on DA (5-HT) neural firing activities between the DRN and VTA populations such that the induced current dynamics could be described by (Wang and Wong-Lin, 2013; Joshi et al., 2017):

$$
\tau_{D A, 5-H T} \frac{d I_{D A, 5-H T}}{d t}=-I_{D A, 5-H T}+\frac{k_{D A, 5-H T}}{1+e^{-g_{D A}\left([5-H T]-[5-H T]_{1}\right)}}
$$




$$
\tau_{5-H T, D A} \frac{d I_{5-H T, D A}}{d t}=-I_{5-H T, D A}+\frac{k_{5-H T, D A}}{1+e^{-g_{5-H T}\left([D A]-[D A]_{1}\right)}}
$$

with the time constants $\tau_{5-H T, D A}=1 \mathrm{~s}$, and $\tau_{D A, 5-H T}=1.2 \mathrm{~s}$ (Aman et al., 2007; Haj-Dahmane, 2001). We set $k_{5-H T, D A}=k_{D A, 5-H T}=0.03$ a.u. and $g_{5-H T}=g_{D A}=20 \mu \mathrm{M}^{-1},[5-H T]_{1}=$ $0.3 \mathrm{nM},[D A]_{1}=0.1 \mathrm{nM}$ such that the neural firing activities and baseline neuromodulator concentration levels were at reasonable values (see below) and similar to those in experiments (e.g. Bunin et al., 1998; Hashemi et al. 2011). For simplicity, we assumed Eqns. (16) and (17) to be applied equally to all targeted neural populations, but with their currents multiplied by their appropriate weights $w^{\prime} s$ (see above).

\section{Release-and-reuptake dynamics of neuromodulators}

The release-and-reuptake dynamics of 5-HT followed the form of a Michaelis-Menten equation (Bunin et al., 1998; Joshi et al., 2011; Hashemi et al., 2011; Joshi et al., 2017):

$$
\frac{d[5-H T]}{d t}=[5-H T]_{p} R_{5-H T}-\frac{V_{\max , 5-H T}[5-H T]}{K_{m, 5-H T}+[5-H T]}
$$

where $[5-H T]_{p}=0.08 \mathrm{nM}$ was defined as the release per firing frequency (Joshi et al., 2011; Flower and Wong-Lin, 2014; Joshi et al., 2017) (value selected to fit to reasonable baseline activities (Hashemi et al. 2011); see below), and the Michaelis-Menten constants $V_{\max , 5-H T}=$ $1.3 \mu \mathrm{M} / \mathrm{s}$ (maximum uptake rate) and $K_{m, 5-H T}=0.17 \mu \mathrm{M}$ (substrate concentration where uptake proceeds at half of maximum rate) were adopted from voltammetry measurements (Hashemi et al. 2011).

Similarly, the release-and-reuptake dynamics for DA was described by

$$
\frac{d[D A]}{d t}=[D A]_{p} R_{D A}-\frac{V_{m a x, D A}[D A]}{K_{m, D A}+[D A]}
$$

where $V_{\max , D A}=-0.004 \mu \mathrm{M} / \mathrm{s}$ and $K_{m, D A}=0.15 \mu \mathrm{M}$ (May et al., 1988). We set $[D A]_{p}=0.1$ $\mathrm{nM}$ to constrain the ratio $[D A]_{p} /[5-H T]_{p}=1.25$ according to May et al. (1988), Bunin et al. (1998), and Hashemi et al. (2011). For simplicity, we assumed Eqns. (18) and (19) to be applied equally to all targeted neural populations.

\section{Reward and punishment conditions with Type-I and Type-II 5-HT neurons}

We focused on only the classical, fully learned reward conditioning task, and unexpected punishment task. For each trial or realisation of simulation within a set of condition (reward/punishment, excitatory/inhibitory connection), we set the cue onset time at $4.5 \mathrm{~s}$ (to allow the network to stabilise substantially). The within-trial protocol for the external input current, $I_{\text {ext }}$, was implemented as a function of time $t$ as followed, depending on the simulated conditions. Note that all external input currents were assumed to be excitatory, regardless of reward or punishment task, unless stated. 
For reward task with Type-I 5-HT neurons: (i) constant input currents $I_{5-H T, \text { ext }}$ and $I_{D A, \text { ext }}$ to 5HT and VTA DA neurons, respectively, of amplitude $50 a$.u. to simulate long-term reward; (ii) brief $0.2 \mathrm{~s}$ pulse $I_{G l u, e x t}$ to DRN Glu neurons of amplitude $1000 \mathrm{a}$.u. at $4.5 \mathrm{~s}$; (iii) constant step input current $I_{G A B A-V T A, \text { ext }}$ to VTA GABAergic neurons with amplitude $200 \mathrm{a}$. u. from 4.5 to 5.7 $\mathrm{s}$ to simulate sustained activity; and (iv) no external input $I_{G A B A-D R N, \text { ext }}$ to DRN GABAergic neurons. For punishment task with Type-I 5-HT neurons: (i) no external input to VTA DA, 5HT neurons and DRN Glu neurons; (ii) brief $0.2 \mathrm{~s}$ pulse to VTA $\left(I_{\text {app }}\right)$ and DRN GABAergic $\left(I_{\text {app } 5 i}\right)$ neurons with amplitude 1000 a.u. at $5.7 \mathrm{~s}$.

For reward task with Type-II 5-HT neurons: (i) constant input current to 5-HT and VTA DA neurons with amplitude 50 a.u. to simulate long-term reward; (ii) additional step input current to 5-HT neurons with amplitude 100 a.u. from 4.5 to $5.7 \mathrm{~s}$ to simulate sustained activity; (iii) brief $0.2 \mathrm{~s}$ pulse to DRN Glu neurons of 1000 a.u. at $4.5 \mathrm{~s}$; (iv) no external input to VTA and DRN GABAergic neurons. For punishment task with Type-II 5-HT neurons: (i) no external input to VTA DA and GABAergic neurons, and DRN Glu neurons; (ii) brief $0.2 \mathrm{~s}$ pulse to DRN GABAergic and 5-HT neurons with amplitude of 1000 a.u. at $5.7 \mathrm{~s}$.

In addition, for transient inputs, we have used multiplicative exponential factors $\exp (-t / \tau)$ with $\tau$ of $50 \mathrm{~ms}$ to smooth out activity timecourses (e.g. afferent synaptic filtering), but they do not affect the overall results. To simulate long-term, across-trial reward/punishment signalling, we assumed a higher constant excitatory input to both 5-HT and DA neurons in reward than punishment trials. When searching for the neural circuit architecture using either Type I or II 5-HT neurons, we limited ourselves as much as possible to the same internal DRN-VTA circuit structure. This also reduced the complexity of the parameter search space.

\section{Baseline neural activities and acceptable deviations}

We define the neural circuit activities under baseline condition (right before cue onset) to follow that in Figs. 3a and 3b. Namely, the baseline firing rates for 5-HT, DRN GABA, DRN GIu, DA and VTA GABA neurons in punishment task were $3.0,21.5,4.1,4.8$ and $13.5 \mathrm{~Hz}$, respectively, and those in reward task were $4.5,19.4,4.1,4.8$ and $16.3 \mathrm{~Hz}$, respectively. The baseline [5 $H T]$ and $[D A]$ levels were constrained to be at 10 and $1.5 \mathrm{nM}$, respectively. However, it is known that these activities can vary widely across subjects, species and studies. Hence, while searching for degenerate neural circuit architecture, we had to define acceptable ranges of neural activities in which we could claim that the variant neural circuit still behaved similarly to that of the model in Fig. 3. Specifically, based on multiple experimental studies (see Supplementary Table 1), we set an inclusion criterion that permitted maximal deviations of the 5-HT, DRN GABA, DRN Glu, DA and VTA GABA neural populations to be less than $10 \%$, $17 \%, 10 \%, 10 \%$ and $17 \%$ from their above defined baseline activities, respectively.

\section{Simulating the effects of $D_{2}$ agonist}

DA and 5-HT induced currents can lead to overall excitatory or inhibitory effects, depending on receptor subtype(s) and the targeted neurons. In particular, DA enhances VTA GABAergic neuronal activity via $\mathrm{D}_{2}$ receptors and depolarizes the membrane of 5-HT neurons (e.g. Ludlow et al., 2009; Haj-Dahmane, 2001; Aman et al., 2007; Courtney et al., 2012; Ford, 2014). DA also regulates the activity of other DA neurons via $D_{2}$ auto-inhibitory receptors (Adell and 
Artigas, 2004). To study how $\mathrm{D}_{2}$ receptor mediated drugs can affect DRN-VTA architecture differently, we simulated different $\mathrm{D}_{2}$ agonist dosage levels simultaneously by multiplying the connection weights (Fig. 4) of $D_{2}$ receptor mediated currents (see above; orange connections in Supplementary Fig. 1) by a factor ' $X$ ' (Fig. 6) of 10, 40, 70 and 100 times. Then for each dosage, we separately observed the deviation in activity profiles for each neural population with respect to the allowed range. Again, we considered the maximal percentage changes due to the drug as significant if the percentage changes in at least one population activity profile were more than the inclusion criterion defined for each population (see above, and Supplementary Tables 1 and 4-8).

\section{Numerical simulations and codes}

Simulations were performed using MATLAB with forward Euler numerical integration method on the dynamical (ordinary differential) equations (see above). A simulation time step of $1 \mathrm{~ms}$ was used and smaller time steps did not affect the results. MATLAB were used for analyses of network stability and sensitivity. Source codes and MATLAB scripts are available upon reasonable request.

\section{Network stability analysis}

The 5 neural population firing rates (Eqns. (4-8)), when combined with their associated afferent currents (Eqns. (9-13)) with explicit parameter values and relative connectivity weights, $W$ 's and J's (Supplementary Fig. 1 and Supplementary Table 2), can be rewritten, respectively, as:

$$
\begin{aligned}
& F_{5-H T}=g_{5-H T}\left[-I_{a u t o, 5-H T}+W_{5 d} I_{5-H T, D A}+J_{55 e} I_{5-H T, G l u}-J_{55 i} I_{5-H T, G A B A-D R N}-\right. \\
& \left.J_{5 i} I_{5-H T, G A B A-V T A}+99.87+I_{5-\mathrm{HT}, \mathrm{ext}}\right]_{+} \\
& F_{D A}=g_{D A}\left[-I_{\text {auto }, D A} \pm W_{d 5} I_{D A, 5-H T}+J_{d 5 e} I_{D A, G l u}-J_{d i} I_{D A, V T A-G A B A}+210+I_{D A, e x t}\right]_{+} \\
& F_{G l u}=g_{G l u}\left[J_{\text {self }, G l u} F_{G l u} \pm W_{5 e 5} I_{G l u, 5-H T}+100+I_{G l u, e x t}\right]_{+} \\
& F_{G A B A-D R N}= \\
& g_{G A B A-D R N}\left[-J_{\text {Self,GABA-DRN }} F_{G A B A-D R N} \pm\right. \\
& W_{5 i 5} I_{G A B A-D R N, 5-H T}+W_{5 i d} I_{G A B A-D R N, D A}-W_{5 i i} I_{G A B A-D R N, G A B A-V T A}+450+ \\
& \left.I_{G A B A-D R N, \text { ext }}\right]_{+} \\
& F_{G A B A-V T A}= \\
& g_{G A B A-V T A}\left[-J_{\text {self,GABA-VTA }} F_{G A B A-V T A}+W_{i 5} I_{G A B A-V T A, 5-H T} \pm W_{i d} I_{G A B A-V T A, D A}+\right. \\
& \left.200+I_{G A B A-V T A, e x t}\right]_{+}
\end{aligned}
$$

We also inserted the explicit parameter values to the dynamical equations (Eqns. (14-19)) to obtain:

$$
500 \frac{d I_{\text {auto }, 5-H T}}{d t}=-I_{\text {auto }, 5-H T}+\frac{80}{1+e^{-10([5-H T]-0.1)}}
$$




$$
\begin{aligned}
150 \frac{d I_{a u t o, D A}}{d t}= & -I_{\text {auto }, D A}+\frac{80}{1+e^{-10([D A]-0.1)}} \\
1200 \frac{d I_{D A, 5-H T}}{d t} & =-I_{D A, 5-H T}+\frac{0.03}{1+e^{-20([5-H T]-0.1)}} \\
1000 \frac{d I_{5-H T, D A}}{d t} & =-I_{5-H T, D A}+\frac{0.03}{1+e^{-20([D A]-0.3)}} \\
\frac{d[5-H T]}{d t} & =\frac{0.08 F_{5-H T}}{1000}-\frac{0.0013[5-H T]}{0.17+[5-H T]} \\
\frac{d[D A]}{d t} & =\frac{0.1 F_{D A}}{1000}-\frac{0.004[D A]}{0.15+[D A]}
\end{aligned}
$$

To check for network stability for each of the considered degenerate neural circuits, we first find each network's steady state (or fixed point) by setting the rate of change for all the dynamical equations to zero, i.e. $\frac{d I_{\text {auto, }, H T}}{d t}=\frac{d I_{\text {auto,DA }}}{d t}=\frac{d I_{D A, 5-H T}}{d t}=\frac{d I_{5-H T, D A}}{d t}=\frac{d[5-H T]}{d t}=$ $\frac{d[D A]}{d t}=0$, and then solving them algebraically. The solution of these equations will give the steady-state value (equilibrium point) of the system. Specifically, the currents (dynamical variables) from Eqns. (25-28) (e.g. $\left.I_{a u t o, 5-H T}=\frac{80}{1+e^{-10([5-H T]-0.1)}}\right)$ were substituted into Eqns. (20-24).

Using only the linear parts of the above threshold-linear functions (which were validated posthoc), and after some algebraic manipulations, we obtained the following system of equations, in matrix form:

$$
\begin{aligned}
& \left(\begin{array}{c}
F_{G l u} \\
F_{G A B A-V T A} \\
F_{G A B A-D R N}
\end{array}\right) \\
& =\left(\begin{array}{ccc}
1-g_{G l u} J_{\text {self }-G l u} & 0 & 0 \\
0 & 1+g_{G A B A-V T A} J_{\text {Self }, G A B A-V T A} & 0 \\
0 & g_{G A B A-D R N} J_{5 i i} & 1+g_{G A B A-D R N} J_{\text {self,GABA-DRN }}
\end{array}\right)^{-1} \\
& \left(\begin{array}{c}
\frac{0.03 W_{5 e 5} g_{G l u}}{1+e^{-20([5-H T]-0.1)}}+\frac{0}{1+e^{-16([D A]-0.1)}}+g_{G l u}\left(100+I_{G l u, e x t}\right) \\
\frac{0.03 W_{i 5} g_{G A B A-V T A}}{1+e^{-20([5-H T]-0.1)}}+\frac{0.03 W_{i d} g_{G A B A-V T A}}{1+a e^{-20([D A]-0.1)}}+g_{G A B A-V T A}\left(200+I_{G A B A-V T A}\right) \\
\frac{0.03 W_{5 i 5} g_{G A B A-D R N}}{1+e^{-20([5-H T]-0.1)}}+\frac{0.03 W_{5 i d} g_{G A B A-D R N}}{1+a e^{-20([D A]-0.1)}}+g_{G A B A-D R N}\left(450+I_{G A B A-D R N}\right)
\end{array}\right) \\
& \left(\begin{array}{c}
F_{5-H T} \\
F_{D A}
\end{array}\right)=\left(\begin{array}{c}
g_{5-H T}\left(\frac{-80}{1+e^{-10([5-H T]-0.1)}}+\frac{0.03 W_{5 d}}{1+a e^{-20([D A]-0.1)}}+99.87+I_{5-H T, \text { ext }}\right) \\
-g_{D A}\left(\frac{0.03 W_{d 5}}{1+e^{-20([5-H T]-0.1)}}+\frac{80}{1+e^{-10([D A]-0.1)}}-210-I_{D A, \text { ext }}\right)
\end{array}\right)+ \\
& \left(\begin{array}{cc}
g_{5-H T} & 0 \\
0 & g_{D A}
\end{array}\right)\left(\begin{array}{ccc}
J_{55 e} & -J_{5 i} & -J_{55 i} \\
J_{d 5 e} & -J_{d i} & 0
\end{array}\right)\left(\begin{array}{c}
F_{G l u} \\
F_{G A B A-V T A} \\
F_{G A B A-D R N}
\end{array}\right)
\end{aligned}
$$

But by setting Eqns. (29) and (30) to be zero, we have, $F_{D A}=\frac{40[D A]}{0.15+[D A]}$ and $F_{5-H T}=\frac{16.25[5-H T]}{0.17+[5-H T]}$ at steady state. Substituting these two into Eqns. (31) and (32), we can solve for the values of $[\mathrm{DA}]$ and $[5-\mathrm{HT}]$ at steady state. 
Next, to check whether the system is stable at the steady state, we compute the 5-by-5 Jacobian matrix $M_{\text {Jacobian }}($ Strogatz, 2018) for the 5 dynamical equations (Eqns. (25-30)), which can be computed using partial derivatives on the right-hand-side of these equations:

$$
\begin{aligned}
& M_{\text {Jacobian }}= \\
& \left(\begin{array}{cccccc}
-1 / 500 & 0 & 0 & 0 & \frac{80}{500} \frac{10 e^{-10([5-H T]-0.1)}}{\left(1+e^{-10([5-H T]-0.1)}\right)^{2}} & \frac{80}{150} \frac{10 e^{-10([D A]-0.1)}}{\left(1+e^{-10([D A]-0.1))^{2}}\right.} \\
0 & -1 / 150 & 0 & 0 & 0 & 0 \\
0 & 0 & -1 / 1200 & 0 & \frac{0.03}{1200} \frac{20 e^{-20([5-H T]-0.1)}}{\left(1+e^{-20([5-H T]-0.1))^{2}}\right.} & \frac{0.03}{1000} \frac{20 e^{-20([D A]-0.3)}}{\left(1+e^{-20([D A]-0.3))^{2}}\right.} \\
0 & 0 & 0 & -1 / 1000 & 0 & 0 \\
\frac{0.008}{1000} g_{5-H T} & 0 & 0 & \frac{0.08}{1000} g_{5-H T} & \frac{-0.0013}{0.17+[5-H T]}+\frac{0.0013[5-H T]}{(0.17+[5-H T])^{2}} & -\frac{0.004}{0.15+[D A]}+\frac{0.004[D A]}{(0.15+[D A])^{2}} \\
0 & \frac{0.1}{1000} g_{D A} & -\frac{0.1}{1000} g_{D A} & 0 & 0 & 0
\end{array}\right.
\end{aligned}
$$

The eigenvalues of this Jacobian matrix were computed for each steady state for each model type under each simulated condition (e.g. reward/punishment task). If the real parts of all the eigenvalues of the Jacobian matrix were negative at a given steady state, then the model was considered to be dynamically stable at that steady state (Strogatz, 2018).

\section{References}

Aman, T. K., Shen, R.-Y. \& Haj-Dahmane, S. D2-like dopamine receptors depolarize dorsal raphe serotonin neurons through the activation of nonselective cationic conductance. $J$. Pharmacol. Exp. Ther. 320(1):376-385 (2007).

Beier, K. T., Steinberg, E. E., DeLoach, K. E., Xie, S., Miyamichi, K., Schwarz, L., Gao. X. J., Kremer, E. J., Malenka, R. C. \& Luo, L. Circuit architecture of VTA dopamine neurons revealed by systematic input-output mapping. Cell 162(3):622-634 (2015).

Benoit-Marand, M., Borrelli, E. \& Gonon, F. Inhibition of dopamine release via presynaptic D2 receptors: Time course and functional characteristics in vivo. J. Neurosci., 21(23):91349141 (2001).

Boureau, Y. L. \& Dayan, P. Opponency revisited: Competition and cooperation between dopamine and serotonin. Neuropsychopharmacology 36(1):74-97 (2011).

Bunin, M. A., Prioleau, C., Mailman, R. B. \& Wightman, R. M. Release and uptake rates of 5hydroxytryptamine in the dorsal raphe and substantia nigra reticulata of the rat brain. $J$. Neurochem. 70(3):1077-1087 (1998).

Canavier, C. C., Evans, R. C., Oster, A. M., Pissadaki, E. K., Drion, G., Kuznetsov, A. S. \& Gutkin, B. S. Implications of cellular models of dopamine neurons for disease. J. Neurophysiol. 116(6):2815-2830 (2016).

Challis, C., Boulden, J., Veerakumar, A., Challis, C., Boulden, J., Veerakumar, A., Espallergues, J., Vassoler, F. M., Pierce, R. C., Beck, S. G. \& Berton, O. Raphe GABAergic neurons mediate the acquisition of avoidance after social defeat. J. Neurosci. 33(35):13978-13988 (2013).

Cohen, J., Haesler, S., Vong, L., Lowell, B. B. \& Uchida, N. Neuron-type specific signals for reward and punishment in the ventral tegmental area. Nature 482(7383):85-88 (2012).

Cohen, J. Y., Amoroso, M. W. \& Uchida, N. Serotonergic neurons signal reward and punishment on multiple timescales. eLife 4:e06346 (2015). doi:10.7554/eLife.06346

Courtney, N. A., Mamaligas, A. A. \& Ford, C. P. Species differences in somatodendritic dopamine transmission determine D2-autoreceptor-mediated inhibition of ventral tegmental area neuron firing. J. Neurosci. 32(39):13520-13528 (2012). 
Crawford, L. K., Craige, C. P. \& Beck, S. G. Increased intrinsic excitability of lateral wing serotonin neurons of the dorsal raphe: A mechanism for selective activation in stress circuits. J. Neurophysiol. 103(5):2652-2663 (2010).

Cropper, E. C., Dacks, A. M. \& Weiss, K. R. Consequences of degeneracy in network function. Curr. Opin. Neurobiol. 41:62-67 (2016).

Cullen, M. \& Wong-Lin, K. Integrated dopaminergic neuronal model with reduced intracellular processes and inhibitory autoreceptors. IET Syst. Biol. 9(6):245-258 (2015).

De Deurwaerdère, P. \& Di Giovanni, G. Serotonergic modulation of the activity of mesencephalic dopaminergic systems: Therapeutic implications. Prog. Neurobiol., 151: 175-236 (2017).

de Jong, J. W., Afjei, S. A., Dorocic, I. P., Peck, J. R., Liu, C., Kim, C. K., Tian, L., Deisseroth \& K., Lammel, S. A neural circuit mechniams for encoding stimuli in the mesolimbic dopamine system. Neuron 101(1):133-151 (2019).

Di Giovanni, G., Di Matteo, V., Pierucci, M. \& Esposito, E. Serotonin-dopamine interaction: electrophysiological evidence. Serotonin-Dopamine Interaction: Experimental Evidence and Therapeutic Relevance. Prog. Brain Res. 172 (2008).

Dorocic, I. P., Furth, D., Xuan, Y., Johansson, Y., Pozzi, L., Silberberg, G., Carlen, M. \& Meletis, K. A whole-brain atlas of inputs to serotonergic neurons of the dorsal and median raphe nuclei. Neuron 83(3):663-678 (2014).

Doya, K. Metalearning and neuromodulation. Neural Netw., 15(4-6):495-506 (2002).

Edelman, G. M. \& Gally, J. A. Degeneracy and complexity in biological systems. Proc. Nat. Acad. Sci. USA 98(24):13763-13768 (2001).

Fernandez, S. P., Cauli, B., Cabezas, C., Muzerelle, A., Poncer, J.-C. \& Gaspar, P. Multiscale single-cell analysis reveals unique phenotypes of raphe $5-\mathrm{HT}$ neurons projecting to the forebrain. Brain Struct. Funct. 221(8):4007-4025 (2016).

Floresco, S. B., West, A. R., Ash, B., Moore, H. \& Grace, A. A. Afferent modulation of dopamine neuron firing differentially regulates tonic and phasic dopamine transmission. Nat. Neurosci. 6(9):968-973 (2003).

Ford, C. P. The role of D2-autoreceptors in regulating dopamine neuron activity and transmission. Neuroscience 282:13-22 (2014).

Haj-Dahmane, S. D2-like dopamine receptor activation excites rat dorsal raphe 5-HT neurons in vitro. Eur. J. Neurosci. 14(1):125-134 (2001).

Hashemi, P., Dankoski, E. C., Wood, K. M., Ambrose, R. E. \& Wightman, R. M. 5-HT and histamine release in the rat substantia nigra pars reticulata following medial forebrain bundle stimulation. J. Neurochem. 118(5):749-759 (2011).

Hayashi, K., Nakao, K. \& Nakamura, K. Appetitive and aversive information coding in the primate dorsal raphé nucleus, J. Neurosci. 31(15):6195-6208 (2015).

Hu, H. Reward and aversion. Annu. Rev. Neurosci. 39:297-324 (2016).

Jalewa, J., Joshi, A., McGinnity, T. M., Prasad, G., Wong-Lin, K. \& Hölscher, C. Neural circuit interactions between the dorsal raphe nucleus and the lateral hypothalamus: An experimental and computational study. PLoS One 9(2):1-16 (2014). doi:10.1371/journal.pone.0088003.

Joshi, A, Wong-Lin, K., McGinnity, T. M. \& Prasad, G. A mathematical model to explore the interdependence between the serotonin and orexin/hypocretin systems. Conf. Proc. IEEE Eng. Med. Biol. Soc. 2011;2011:7270-7273 (2011).

Joshi, A., Youssofzadeh, V., Vemana, V., McGinnity, T. M., Prasad, G. \& Wong-Lin, K. An integrated modelling framework for neural circuits with multiple neuromodulators. J. $R$. Soc. Interface 14(126):20160902 (2017). doi:10.1098/rsif.2016.0902.

Kaczmarek, L. K. \& Levitan, I. B. Neuromodulation: The biochemical control of neuronal excitability. New York: Oxford University Press (1987).

Lammel, S., Lim, B.K. \& Malenka, R.C. Reward and aversion in a heterogeneous midbrain dopamine system. Neuropharmacology 76 Pt B (2014):351-359 (2014). doi:10.1016/j.neuropharm.2013.03.019.

Li, Y., Zhong, W., Wang, D., Feng, Q., Liu, Z., Zhou, J., Jia, C., Hu, F., Zeng, J., Guo, Q., Fu \& L., Luo, M. Serotonin neurons in the dorsal raphe nucleus encode reward signals. Nat. 
Commun. 7:10503 (2016). https://doi.org/10.1038/ncomms10503.

Li, Y., Li, C. Y., Xi, W., Jin, S., Wu, Z. H., Jiang, P., Dong, P., He, X. B., Xu, F. Q., Duan, S., Zhou, Y. D. \& Li, X. M. Rostral and caudal ventral area GABAergic inputs to different dorsal raphe neurons participate in opioid dependence. Neuron, 101(4):748-761 (2019).

Liu, Z., Zhou, J., Li, Y., Hu, F., Lu, Y., Feng, Q., Zhang, J.-E., Wang, D., Zeng, J., Bao, J., Kim, J.Y., Chen, Z.-F., Mestikaway, S. E. \& Luo, M. Dorsal raphe neurons signal reward through 5-HT and glutamate, Neuron 81(6):1360-1374 (2014).

Ludlow, K. H., Bradley, K. D., Allison, D. W., Taylor, S. R., Yorgason, J. T., Hansen, D. M., Walton, C. H., Sudweeks, S. N. \& Steffensen, S. C. Acute and chronic ethanol modulate dopamine D2-subtype receptor responses in ventral tegmental area GABA neurons. Alcohol Clin. Exp. Res. 33(5):804-811 (2009).

Marder, E. Neuromodulation of neuronal circuits: back to the future. Neuron 76(1):1-11 (2012).

Marder, E., O'Leary, T. \& Shruti, S. Neuromodulation of circuits with variable parameters: single neurons and small circuits reveal principles of state-dependent and robust neuromodulation. Annu. Rev. Neurosci. 37:329-346 (2014).

Matias, S., Lottem, E., Dugué, G. P. \& Mainen, Z. F. Activity patterns of serotonin neurons underyling cognitive flexibility. eLife 6:e20552 (2017). doi:10.7554/eLife.20552

May, L. J., Kuhr, W. G. \& Wightman, R. M. Differentiation of dopamine overflow and uptake processes in the extracellular fluid of the rat caudate nucleus with fast-scan in vivo voltammetry. J. Neurochem. 51(4):1060-1069 (1988).

McDevitt, R. A., Tiran-Cappello, A., Shen, H., Balderas, I., Britt, J. P., Marino, R. A. M., Chung, S. L., Richie, C. T., Harvey \& B. K., Bonci, A. Serotonergic versus non-serotonergic dorsal raphe projection neurons: differential participation in reward circuitry. Cell Rep. 8(6):1857-1869 (2014).

Müller, C.P. \& Cunningham, K.A. Handbook of the Behavioral Neurobiology Serotonin. Academic Press (2020).

Ogawa, S. K., Cohen, J. Y., Hwang, D., Uchida, N. \& Watabe-Uchida, M. Organization of monosynaptic inputs to the serotonin and dopamine neuromodulatory systems. Cell Rep. 8:1105-1118 (2014).

Ogawa, S. K. \& Watabe-Uchida, M. Organization of dopamine and serotonin system: Anatomical and functional mapping of monosynaptic inputs using rabies virus. Pharmacol. Biochem. Behav. 174:9-22 (2018).

Okaty, B. W., Commons, K. G. \& Dymecki, S. M. Embracing diversity in the 5-HT neuronal system. Nat. Rev. Neurosci. 20(7):397-424 (2019).

Ren, J., Friedman, D., Xiong, J., Liu, C. D., Ferguson, B. R., Weerakkody, T., DeLoach, K. E., Ran, C., Pun, A., Sun, Y., Weissbourd, B., Neve, R. L., Huguenard, J., Horowitz, M. A. \& Luo, L. Anatomically defined and functionally distinct dorsal raphe serotonin subsystems. Cell 175(2):472-487 (2018).

Richards, C. D., Shiroyama, T. \& Kitai, S. T. Electrophysiological and immunocytochemical characterization of GABA and dopamine neurons in the substantia nigra of the rat. Neuroscience 80(2):545-557 (1997).

Ritter, J., Lewis, L., Mant, T. \& Ferro, A. A textbook of clinical pharmacology and therapeutics. CRC Press (2008).

Schultz, W., Dayan \& P., Montague, P. R. A neural substrate of prediction and reward. Science 275(5306):1593-1599 (1997).

Shepard, P. D. \& Bunney, B. S. Repetitive firing properties of putative dopamine-containing neurons in vitro: regulation by an apamin-sensitive $\mathrm{Ca}(2+)$-activated $\mathrm{K}+$ conductance. Exp. Brain Res. 86(1):141-50 (1991).

Strogatz, S. H. Nonlinear dynamics and chaos: With applications to physics, biology, chemistry, and engineering. CRC Press (2018).

Tan, K. R., Yvon, C., Turiault, M., Mirzabekov, J. J., Doehner, J., Labouèbe, G., Deisseroth, K., Tye, K. M. \& Lüscher, C. GABA neurons of the VTA drive conditioned place aversion. Neuron 73(6):1173-1183 (2012).

Tian, J., Huang, R., Cohen, J. Y., Osakada, F., Kobak, D., Machens, C. K., Callaway, E. M., 
Uchida, N. \& Watabe-Uchida, M. Distributed and mixed information in monosynaptic inputs to dopamine neurons. Neuron 91:1374-1389 (2016).

Tuckwell, H. C. \& Penington, N. J. Computational modeling of spike generation in serotonergic neurons of the dorsal raphe nucleus. Prog. Neurobiol. 118:59-101 (2014).

Valencia-Torres, L., Olarte-Sanchez, C. M., Lyons, D. J., Georgescu, T., Greenwald-Yarnell, M., Myers Jr., M. G., Bradshaw, C. M. \& Heisler, L. K. Activation of ventral tegmental area 5-HT2C receptors reduces incentive motivation. Neuropsychopharmacology 42(7):1511-1521 (2017).

Wang, D.-H. \& Wong-Lin, K. Comodulation of dopamine and serotonin on prefrontal cortical rhythms: A theoretical study. Front. Integr. Neurosci. 7:54 (2013). doi:10.3389/fnint.2013.00054.

Wang, H. L., Zhang, S., Qi, J., Wang, H., Cachope, R., Mejias-Aponte, C. A., Gomez, J. A., Mateo-Semidey, G. E., Beaudoin, G. M. J., Paladini, C. A., Cheer, J. F. \& Morales, M. Dorsal raphe dual serotonin-glutamate neurons drive reward by establishing excitatory synapses on VTA mesoaccumbens dopamine neurons. Cell Rep. 26(5):1128-1142 (2019).

Watabe-Uchida, M., Zhu, L., Ogawa, S. K., Vamanrao, A. \& Uchida, N. Whole-brain mapping of direct inputs to midbrain dopamine neurons. Neuron 74:858-873 (2012).

Watabe-Uchida, M., Eschel, N. \& Uchida, N. Neural circuitry of reward prediction error. Annu. Rev. Neurosci. 40:373-394 (2017).

Weissbourd, B., Ren, J., DeLoach, K. E., Guenthner, C. J., Miyamichi, K. \& Luo, L. Presynaptic partners of dorsal raphe serotonergic and GABAergic neurons. Neuron 83:645-662 (2014).

Whitacre, J. M. Degeneracy: a link between evolvability, robustness and complexity in biological systems. Theor. Biol. Med. Model. 7:6 (2010). doi:10.1186/1742-4682-7-6.

Wong-Lin, K., Joshi, A., Prasad, G. \& McGinnity, T. M. Network properties of a computational model of the dorsal raphe nucleus. Neural Netw. 32:15-25 (2012).

Wong-Lin, K., Wang, D.-H., Moustafa, A. A., Cohen, J. Y. \& Nakamura, K. Toward a multiscale modeling framework for understanding serotonergic function. J. Psychopharmacol. 31(9):1121-1136 (2017).

Xu, P., He, Y., Cao, X., Valencia-Torres, L., Yan, X., Saito, K., Wang, C., Yang, Y., Hinton Jr., A., Zhu, L., Shu, G., Myers Jr., M. G., Wu, Q., Tong, Q., Heisler \& Xu, Y. Activation of serotonin $2 \mathrm{C}$ receptors in dopamine neurons inhibits binge-like eating in mice. Biol. Psychiatry, 81(9):737-747 (2017).

Yamaguchi, T., Qi, J., Wang, H.-L., Zhang, S. \& Morales, M. Glutamatergic and dopaminergic neurons in the mouse ventral tegmental area. Eur. J. Neurosci. 41(6):760-772 (2015).

Zhong, W., Li, Y., Feng, Q. \& Luo, M. (2017) Learning and stress shape the reward response patterns of serotonin neurons. J. Neurosci. 37(37):8863-8875.

Zhou, L. Liu, M.-Z., Li, Q., Deng, J., Mu, D. \& Sun, Y.-G. Organization of functional long-range circuits controlling the activity of serotongergic neurons in the dorsal raphe nucleus. Cell Rep. 18:3018-3032 (2017).

Zhou, H., Wong-Lin \& K., Wang, D.-H. Parallel excitatory and inhibitory neural circuit pathways underlie reward-based phasic neural responses. Complexity, 2018:4356767 (2018). doi:10.1155/2018/4356767. 